\title{
Metabolomic biomarkers of polycystic ovary syndrome related-obesity: a review of the literature
}

\author{
Camelia Larisa Vonica ${ }^{1,2 \#}$, Anca Daniela Farcas ${ }^{3 \#}$, Gabriela Roman ${ }^{1}$, Andrada \\ Alina Muresan ${ }^{1}$, Adriana Fodor $^{1 *}$, Simona Cernea ${ }^{4,5}$,Carmen Emanuela \\ Georgescu $^{2}$
}

1. "Iuliu Hatieganu" University of Medicine and Pharmacy, 6th Department of Medical specialities, Diabetes and Nutrition Diseases, Cluj-Napoca, Romania

2. "Iuliu Hatieganu" University of Medicine and Pharmacy, 6th Department of Medical specialities Endocrinology, Cluj-Napoca, Romania

3. "Iuliu Hatieganu” University of Medicine and Pharmacy, Department of Cardiology and Internal Medicine I, Cluj-Napoca, Romania

4. Faculty of Medicine/Department M4, George Emil Palade University of Medicine, Pharmacy, Science, and Technology of TarguMures, Targu Mures, Romania

5 Diabetes, Nutrition and Metabolic Diseases Outpatient Unit, Emergency County Clinical Hospital, Targu Mures, Romania

\begin{abstract}
Background and objectives: Polycystic ovary syndrome (PCOS) displays a phenotype-dependent cardio-metabolic risk. By performing a systematic search of the literature, we aimed to summarize metabolomic signatures associated with obesity in PCOS women. Data sources and study eligibility criteria: We conducted a comprehensive search including: Embase, PubMed, and Web of Science until 31st of May 2019. We used the terms: metabolomics and polycystic ovary syndrome. We excluded the following papers: animal studies, studies that included only lean PCOS women, reviews, meta-analyses, results of interventional studies, those that did not apply metabolomic techniques. Results: The lipid signature in obese women with PCOS showed increased levels of free fatty acids (carnitine, adipic acid, linoleic acid, oleic acid) and lower levels of lysophosphatidylcholines and glycerolphosphocholine compared with non-obese PCOS women. Regarding carbohydrate metabolism, a decrease in citric and lactic acid levels characterized obese PCOS women. Decreased lactic acid in obese PCOS women suggests augmented insulin stimulated glucose muscle use in lean, but not in obese women. Considering amino acid metabolomic markers, valine, glycine, serine, threonine, isoleucine and lysine were higher in obese PCOS women. Patients with visceral
\end{abstract}

*Corresponding author: Adriana Fodor, "Iuiu Hatieganu” University of Medicine and Pharmacy, 6th Department of Medical specialities, Diabetes and Nutrition Diseases, Cluj-Napoca, Romania. E-mail: adifodor@yahoo.com \# Authors with equal contribution 
obesity presented a diminished uptake of essential amino acids, BCAA, leucine and serine in the skeletal muscle. $\alpha$-ketoglutarate was significantly higher in obese women with PCOS in comparison with lean women with PCOS, distinguishing these 2 subgroups of PCOS with high 'predictive accuracy'. Limitations: Overall, a small number of studies have focused on the impact of obesity on the metabolic fingerprints of PCOS women. There is need for properly controlled, high-quality studies. Conclusions: There is compelling evidence of significant alterations in carbohydrate, lipid, and amino acid metabolism in women with PCOS and obesity. Metabolomics may identify new metabolic pathways involved in PCOS and improve our understanding of the complex relation between PCOS and obesity in order to personalize PCOS therapy.

Keywords: untargeted metabolomics, polycystic ovary syndrome, obesity, biomarkers, targeted metabolomics Received: $9^{\text {th }}$ December 2019; Accepted: $13^{\text {th }}$ February 2020; Published: $24^{\text {th }}$ February 2020

\section{Introduction}

\section{Rationale and background}

Polycystic ovary syndrome (PCOS) is a heterogenous disease affecting between 6 to $20 \%$ of women at reproductive age, with a variable cardio-metabolic risk (1). Obesity and insulin resistance (IR) are probably the most common (about 50\%, and 70\% respectively) and important features of PCOS $(2,3)$. However, they are not part of the diagnostic criteria $(2,3)$. Androgen excess, IR and obesity, mainly the android phenotype, variably contribute to chronic lowgrade inflammation and cardio-metabolic risk in obese PCOS women (1). A recent meta-analysis which included 29 studies showed that women with PCOS are at higher risk of developing obstetrical complications like miscarriages, pregnancy-induced hypertension, gestational diabetes mellitus, large-for-gestational-age babies and preterm birth (4).

Up-to-date research is progressively focusing on the discovery of innovative biomarkers to better understand the metabolic changes in PCOS. (5) A step forward was made in recent years by metabolomics technology, aiming to identify metabolites that represent chemical fingerprints of various metabolic pathways relevant for the pathogenesis of PCOS. Metabolomic profiling in PCOS has made its first steps in the early 2012, with few studies that focused on characterization of the metabolomic signature of lipid metabo- lism, hormone biosynthesis, medical therapies, and assisted reproduction outcomes (6-10).

Objectives: By performing a systematic search of the literature, we aimed to summarize metabolomic pathways and biomarkers associated with obesity in women with PCOS.

\section{Analytical Techniques in Metabolomics}

Metabolomics is a bio-analytical technique used for the identification and quantification of metabolites in various biological samples, that allows the characterization of the individual metabolomic fingerprint. This technique is also used to decipher the complex metabolic fluctuations that occur in various clinical conditions (11). The use of mass spectrometry (MS)-based techniques for metabolomics analysis shows high sensitivity, especially when MS is used as liquid chromatography (LC) (LC-MS) (12). This type of metabolomics analysis allows quantitative assessment in the picomolar ranges (12). Easy sample preparation and reproducibility favor the use of nuclear magnetic resonance (NMR) as an analytical method. (13). NMR is a spectroscopic technique used for metabolomics determination. Its principles are based on the fact that atomic nuclei have magnetic properties and their behavior in a magnetic field offers information about the molecule regarding its structural and chemical properties (13).

Gas chromatography-mass spectrometry (GC$\mathrm{MS}$ ) is a double component system that uses GC 
to separate volatile mixtures of compounds, and concomitantly uses MS to detect the eluted compounds. GC-MS has a high sensitivity (picomolar range versus micromolar range for NMR) for identifying different types of metabolites like organic acids, amino acids, aromatic amines, fatty acids, and sugars (12).

There are two types of metabolomic analyses: targeted, which identifies and quantifies selected metabolites from a biological sample, and untargeted, which measures all the metabolites in a biological sample. However, the second type emerges as a useful tool in detecting new biomarkers of diagnosis and prognosis (12). Nevertheless, before translating a potential metabolite biomarker into clinical use, additional validation studies are required (14).

\section{Methods}

In order to achieve the objectives of this review, we designed a protocol following the Preferred Reporting Items for Systematic Reviews and Meta-Analyses (PRISMA) protocol (15).

\section{Literature search}

We conducted a comprehensive search including: Embase, PubMed, and Web of Science until $31^{\text {st }}$ of May 2019; using the search terms: metabolomics and polycystic ovary syndrome. An example of the search query used in one of the databases is provided below:

(("metabolomics"] [MeSH Terms] OR "metabolomics"[All Fields]) AND ("polycystic ovary syndrome"[MeSH Terms] OR ("polycystic" [All Fields] AND "ovary"[All Fields] AND "syndrome"[All Fields]) OR "polycystic ovary syndrome"[AllFields])AND("0001/01/01" [PDAT] : “2019/05/31" [PDAT]))

We aimed to identify all relevant studies regardless of publication status (published, unpublished, in press, or in progress). This initial search yielded 213 articles (Figure 1).
After excluding duplicates, two independent reviewers selected eligible articles by reading titles and abstracts of the remaining articles. Fulltext copies of articles considered eligible in this step of the review were evaluated by the two authors for inclusion and exclusion criteria. A third reviewer participated in this process in order to make an objective decision whenever a disagreement surfaced between them.

\section{Inclusion and exclusion criteria}

We included all cross-sectional studies, observational studies, and baseline results of interventional trials that were performed in adult $(>18$ years of age) females with PCOS and in whom PCOS was diagnosed by Rotterdam criteria.

We excluded studies published in languages other than English, commentaries/personal opinions, review articles, meta-analyses and book chapters, studies which enrolled children or adolescents, in vitro or in vivo animal studies, included only lean PCOS women, did not apply metabolomic techniques.

\section{Data extraction and synthesis}

After the selection process, data were extracted by two independent reviewers following an excel extraction form. We extracted the following information: author, year of publication, study design, sample size, type of study design, selection criteria, type of metabolomic technique used (i.e. NMR or MS), type of sample used (urine, blood, saliva, follicular fluid), and a list of metabolites identified to be altered in obese PCOS women versus lean PCOS/ obese controls/ lean controls (Tables 1 and 2).

\section{Outcome measures}

The main outcome measures of included studies in the systematic review were:

- Carbohydrate metabolism in obese PCOS

- Lipid metabolism in PCOS in relation to obesity 


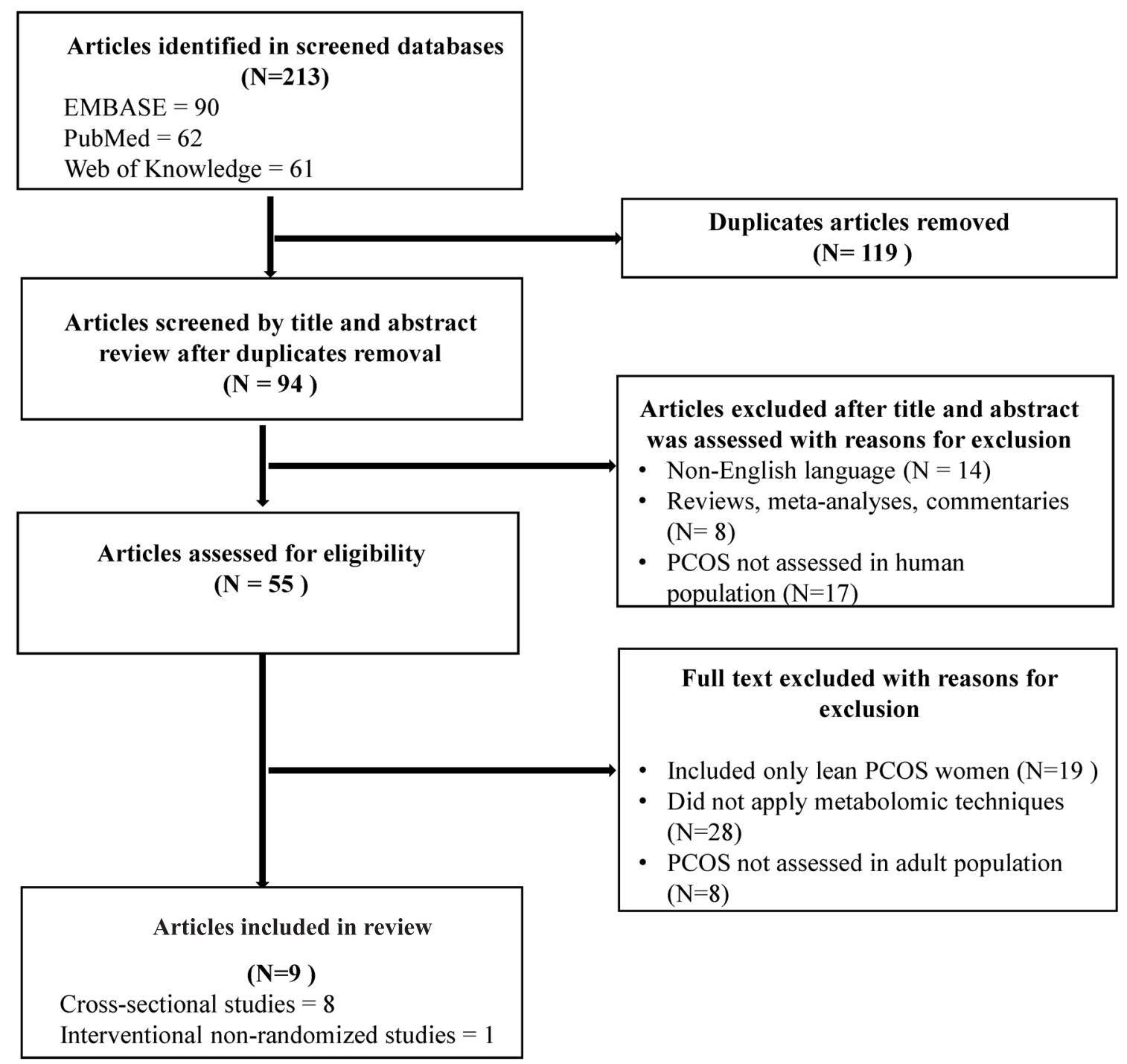

Fig. 1. A flow chart summarizing the selection process

- Amino acid metabolisms in PCOS and obesity

- Alterations of the tricarboxylic acid cycle (TCA) metabolism in obese PCOS women

- Steroid metabolites profiling in patients with PCOS and obesity.

\section{Quality assessment and risk of bias}

In order to assess quality of the prospective ob- servational studies we used Methodological index for non-randomized studies; all studies with a score of $\geq 12$ were included in this review (16). Regarding randomized clinical trials, we assessed the risk of bias by using the Cochrane risk of bias tool (17). We included only the randomized clinical trials graded as fair or good quality. As for cross-sectional studies, the Quality Assessment Tool of the National Heart, Lung, and 
Blood Institute was used (18). We included only those graded as fair or good quality.

\section{Results}

\section{Study selection}

The initial search of the above mentioned databases yielded 213 results. After we removed the duplicates, 94 underwent title and abstract screening for the inclusion/exclusion criteria. Subsequently, 55 articles underwent full-text review, and 46 were excluded because they did not meet the inclusion/exclusion criteria, due to quality issues, did not apply metabolomics or included only lean PCOS women. Thus, we included 9 studies in this systematic review (Figure 1).

\section{Carbohydrate metabolism in obese PCOS}

GC-MS-based metabolomics conducted by Escobar-Morreale et al. and recently by Troisi et al. identified increased levels of lactic acid in lean and overweight PCOS women and decreased levels in obese PCOS women, suggesting an augmented muscle insulin-stimulated glucose use in lean, but not obese women with PCOS $(19,20)$. This difference may indicate that IR might be more severe and extensive in obese patients $(19,20)$.

A diminished ability to switch from carbohydrate or protein to lipid use in periods of fasting, including overnight fast, has been reported as a metabolic defect associated with obesity, IR and PCOS $(21,22,23)$. It was reported that upon stimulation with $75 \mathrm{~g}$ of oral glucose after an overnight fast, there is a decrease in pyruvate, alanine, lactate, and acetate levels and an increase in fructose and glucose levels in PCOS patients compared with healthy subjects (24). In conclusion, women with PCOS may exhibit a diminished ability to switch from glucose or amino acid to lipid oxidation during fasting periods. This metabolic inflexibility seems to con- tribute to the progressive obesity that commonly accompanies PCOS (24).

\section{The lipid signature in PCOS in relation to obesity}

By using a nontargeted metabolomics approach, Zhao X. et al. found increased serum levels of total free fatty acids (FFAs) in subjects with PCOS when compared with control subjects, particularly in overweight PCOS (25). The increased levels of circulating FFAs may in turn contribute to adverse metabolic traits of PCOS, including IR, type 2 diabetes mellitus, arterial hypertension, dyslipidemia, etc. $(26,27)$. When the authors looked at the FFAs pattern, they found a percentage increase in monounsaturated fatty acid (MUFA) and percentage decrease in saturated fatty acid (SFA) in both lean and overweight PCOS (25). However, the ratio of MUFA to SFA species, as a measure of stearoyl-CoA desaturase activity (SCD) (28), increased significantly more in obese compared with lean PCOS patients. The SCD activity was previously reported to be closely related to cholesterol intake, hormonal imbalance, body adiposity, etc. (2931). Also, increased SCD activity has been associated with increased risk of cardiometabolic diseases, neurological diseases and cancer (28, 31, 32). Consequently, altered FFAs metabolism involving increased SCD activity may be closely related to PCOS comorbidities.

Likewise, differences between subgroups in relation to obesity were recorded with regards to lysophosphatidylcholines (LPC) (25). LPC are a class of phospholipids that may induce an inflammatory response, but also play an important role in glucose metabolism and contribute to IR $(33,34)$. Several authors reported a plasma longchain fatty acids (linoleic, oleic acid, glycerol) excess in overweight/obese patients with PCOS, as well as increased levels of oleic and palmitoleic acid in obese women, irrespective of the presence of PCOS $(19,20,35)$. They demon- 


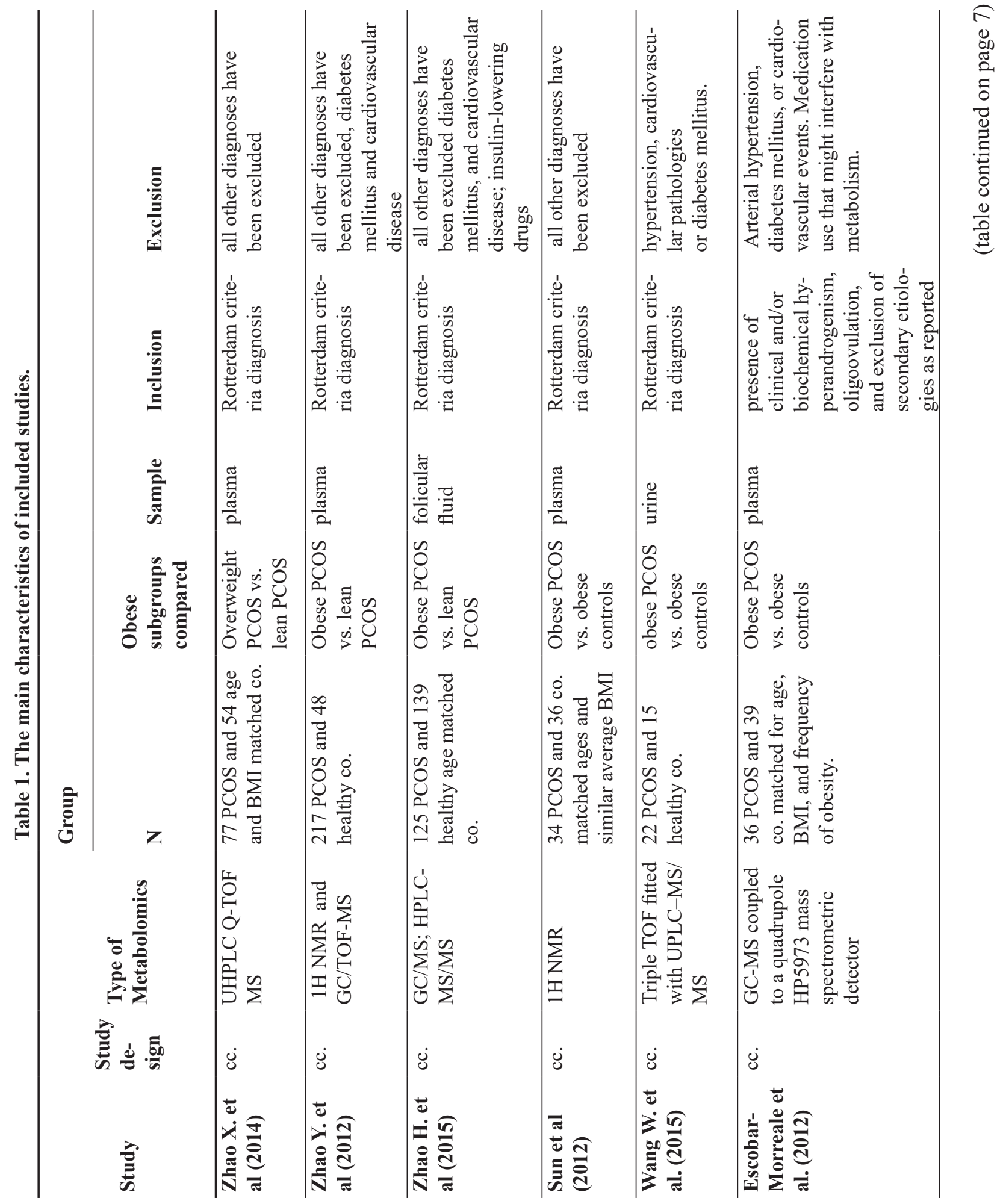




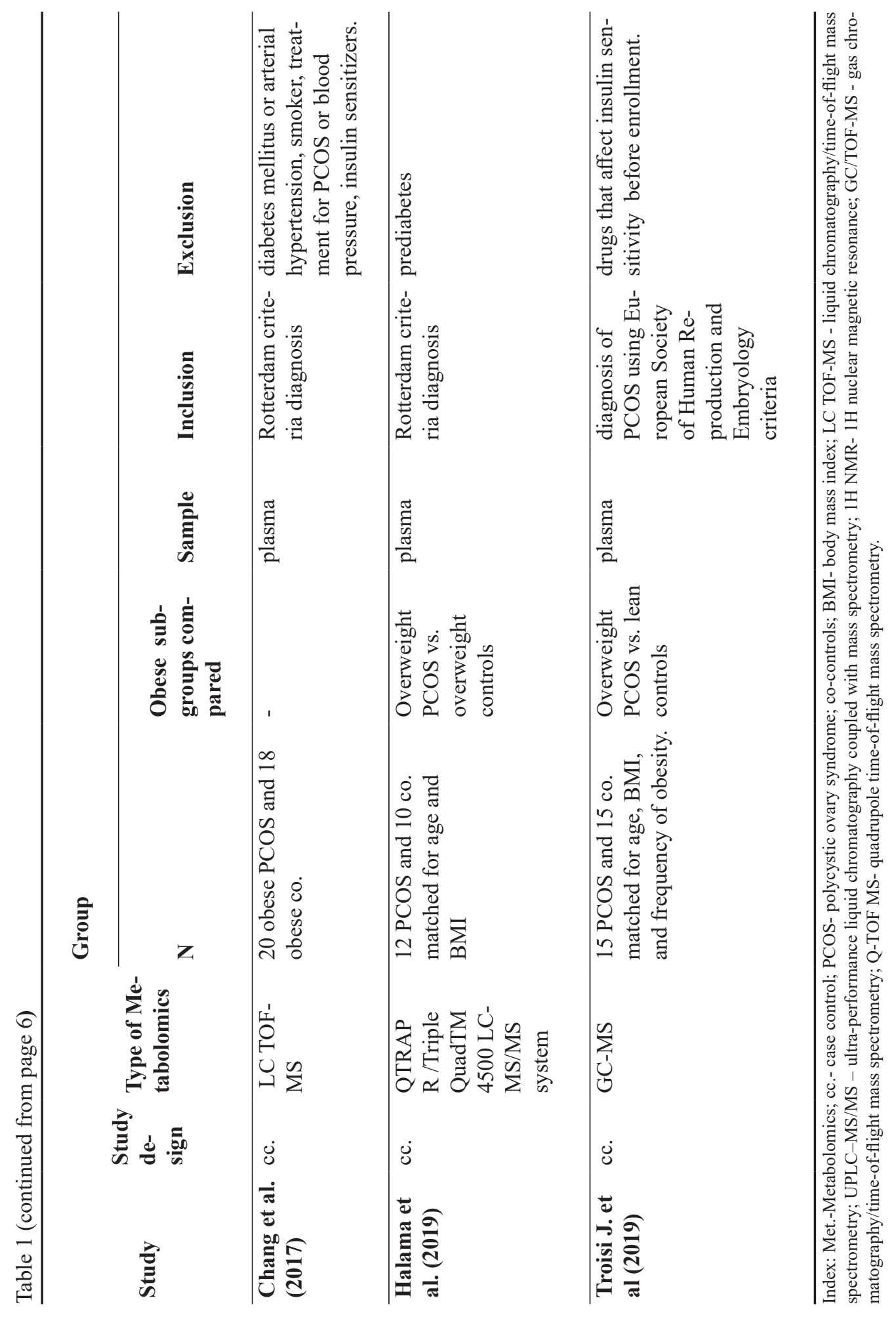


Table 2. The metabolites identified in obese PCOS women vs. lean PCOS women or obese controls.

\begin{tabular}{|c|c|c|c|c|}
\hline Authors & Compared groups & Metabolite & Tendency & Signaled pathway \\
\hline \multirow[t]{14}{*}{$\begin{array}{l}\text { Zhao X. et al } \\
2014\end{array}$} & $\begin{array}{c}\text { Overweight PCOS vs. } \\
\text { lean PCOS }\end{array}$ & & & \\
\hline & & Carnitine C18:1 & $\uparrow$ & Lipid metabolism \\
\hline & & FFA 16:1 & $\uparrow$ & Lipid metabolism \\
\hline & & FFA 18:1 & $\uparrow$ & Lipid metabolism \\
\hline & & FFA 20:3 & $\uparrow$ & Lipid metabolism \\
\hline & & FFA 20:4 & $\uparrow$ & Lipid metabolism \\
\hline & & FFA 20:5 & $\uparrow$ & Lipid metabolism \\
\hline & & FFA 22:5 & $\uparrow$ & Lipid metabolism \\
\hline & & LPC 18:1 & $\downarrow$ & Lipid metabolism \\
\hline & & LPC 18:2 & $\downarrow$ & Lipid metabolism \\
\hline & & LPC 20:5 & $\downarrow$ & Lipid metabolism \\
\hline & & Gly.Phe & $\uparrow$ & AAA metabolism \\
\hline & & Phenylalanine & $\uparrow$ & AAA metabolism \\
\hline & & Phe-Phe & $\uparrow$ & AAA metabolism \\
\hline \multirow[t]{4}{*}{$\begin{array}{l}\text { Zhao Y. et al } \\
2012\end{array}$} & $\begin{array}{c}\text { Obese PCOS vs. lean } \\
\text { PCOS }\end{array}$ & Valine & $\uparrow$ & AA metabolism \\
\hline & & Glycine & $\uparrow$ & AA metabolism \\
\hline & & Serine & $\uparrow$ & AA metabolism \\
\hline & & Threonine & $\uparrow$ & AA metabolism \\
\hline \multirow[t]{3}{*}{$\begin{array}{l}\text { Zhao H. et al } \\
2015\end{array}$} & $\begin{array}{c}\text { Obese PCOS vs. lean } \\
\text { PCOS }\end{array}$ & $\alpha$ - ketoglutarate & $\uparrow$ & Carbohydrate metabolism \\
\hline & & Valine & $\uparrow$ & BCAA metabolism \\
\hline & & Leucine & $\uparrow$ & BCAA metabolism \\
\hline \multirow[t]{2}{*}{$\begin{array}{l}\text { Sun L. et al } \\
2012\end{array}$} & $\begin{array}{l}\text { Obese PCOS vs. } \\
\text { obese controls }\end{array}$ & Citrate & $\downarrow$ & Carbohydrate metabolism \\
\hline & & $\begin{array}{c}\text { Glycerophosphocholine/ } \\
\text { phosphocholine }\end{array}$ & $\downarrow$ & Lipid metabolism \\
\hline \multirow[t]{3}{*}{$\begin{array}{l}\text { Sun L. et al } \\
2012\end{array}$} & $\begin{array}{l}\text { Obese PCOS vs. } \\
\text { obese controls }\end{array}$ & Citrate & $\downarrow$ & Carbohydrate metabolism \\
\hline & & $\begin{array}{l}\text { Glycerophosphocholine/ } \\
\text { phosphocholine }\end{array}$ & $\downarrow$ & Lipid metabolism \\
\hline & & Leucine & $\downarrow$ & BCAA metabolism \\
\hline \multirow[t]{3}{*}{$\begin{array}{l}\text { Wang W. et al } \\
2015\end{array}$} & $\begin{array}{c}\text { Obese PCOS vs. } \\
\text { obese controls }\end{array}$ & Cortolone-3-glucuronide & $\uparrow$ & Lipid metabolism \\
\hline & & $\begin{array}{l}\text { Tetrahydroaldoste- } \\
\text { rone-3-glucuronide }\end{array}$ & $\uparrow$ & Lipid metabolism \\
\hline & & Testosterone glucuronide & $\uparrow$ & Steroid metabolism \\
\hline \multirow[t]{2}{*}{$\begin{array}{l}\text { Escobar-Mor- } \\
\text { reale H. et al } \\
2012 \\
\end{array}$} & $\begin{array}{l}\text { Obese PCOS vs. } \\
\text { obese controls }\end{array}$ & Lactic acid & $\downarrow$ & Carbohydrate metabolism \\
\hline & & Adipic acid & $\uparrow \uparrow$ & Lipid metabolism \\
\hline
\end{tabular}


(table continued from page 8)

\begin{tabular}{lcccc}
\hline Authors & Compared groups & Metabolite & Tendency & Signaled pathway \\
\hline & Glyceric acid & $\uparrow$ & Lipid metabolism \\
\hline & Glycerol & $\uparrow$ & Lipid metabolism \\
\hline Chang Y. et al & Linoleic acid & $\uparrow$ & Lipid metabolism \\
\hline $\mathbf{2 0 1 7}$ & Obese PCOS vs. & $\uparrow$ & Lipid metabolism \\
\hline & obese controls & Linoleic acid & $\uparrow$ & Lipid metabolism \\
\hline & Lysine & $\uparrow$ & AA metabolism \\
\hline & $\alpha$-aminoadipic acid & $\uparrow$ & AA metabolism \\
\hline & Tyrosine & $\uparrow$ & AAA metabolism \\
\hline Halama et al. & Valine & $\uparrow$ & BCAA metabolism \\
\hline Ov19 & Isoleucine & $\uparrow$ & BCAA metabolism \\
\hline & Leucine & $\uparrow$ & BCAA metabolism \\
\hline & Lerweight PCOS vs. & $\uparrow$ cine/Isoleucine & $\uparrow$ & AA metabolism \\
\hline & Glutamate & $\uparrow$ & AA metabolism \\
\hline & Methionine & $\uparrow$ & AA metabolism \\
\hline & Ornithine & $\uparrow$ & AA metabolism \\
\hline & Phenylalanine & $\uparrow$ & AA metabolism \\
\hline & Tyrosine & $\uparrow$ & AA metabolism \\
\hline & Proline & $\uparrow$ & AA metabolism \\
\hline
\end{tabular}

\begin{tabular}{|c|c|c|c|c|}
\hline $\begin{array}{l}\text { Troisi J. et al } \\
2019\end{array}$ & $\begin{array}{c}\text { Overweight PCOS vs. } \\
\text { lean controls }\end{array}$ & Lactic acid & $\uparrow$ & Carbohydrate metabolism \\
\hline & & Glucose & $\uparrow$ & Carbohydrate metabolism \\
\hline & & Linoleic acid & $\uparrow$ & Lipid metabolism \\
\hline & & Palmitic acid & $\uparrow$ & Lipid metabolism \\
\hline & & Glyceric acid & $\downarrow$ & Lipid metabolism \\
\hline & & Valine & $\uparrow$ & TCA \\
\hline & & Phenylalanine & $\uparrow$ & TCA \\
\hline & & Glutamine & $\downarrow$ & TCA \\
\hline & & Arginine & $\downarrow$ & TCA \\
\hline & & Citric acid & $\downarrow$ & TCA \\
\hline & & Choline & $\downarrow$ & TCA \\
\hline & & Ketoisocapric acid & $\uparrow$ & AA metabolism \\
\hline & & Creatinine & $\downarrow$ & AA metabolism \\
\hline
\end{tabular}


strated that obese patients with PCOS had a different profile of FFA metabolites suggestive of increased lipolysis compared with non-obese women with PCOS, who demonstrated suppression of lipolysis. Accelerated lipolysis is possibly due to impaired insulin action in adipose tissue (19). Nonetheless, this vicious circle further promotes IR when PCOS is associated with obesity (36). This triad of metabolic impairments is further linked to metabolic syndrome and non-alcoholic fatty liver disease (NAFLD) (37).

Moreover, two studies showed that subjects with PCOS have a pro-atherogenic lipid profile characterized by higher levels of triglycerides and LDL-cholesterol (LDL), and lower levels of HDL-cholesterol (HDL) $(26,38)$.

\section{Amino acid metabolisms in PCOS and obe- sity}

Chang et al. compared obese PCOS women with age- and BMI-matched controls without PCOS (35). They observed higher concentrations of branched-chain amino acids (BCAA), essential amino acids, and lysine metabolite $\alpha$-amino-acids (AA) in obese PCOS compared with control obese patients. The authors suggest that IR, but not obesity or hyperandrogenism is responsible for the metabolic changes in PCOS (35). On the other hand, Zhao et al. also showed that the levels of valine and leucine were significantly increased in the obese subgroup of women with PCOS compared with non-obese PCOS women, while isoleucine and intermediates of BCAA catabolism did not differ between the two subgroups (27).

When the metabolomic pattern of visceral and subcutaneous adipose tissue samples was analyzed in non-obese and obese subjects, patients with visceral obesity presented a diminished uptake of essential amino acids, BCAA, leucine, and serine. Furthermore, these metabolic alterations appeared before any significant clinical onset (39). Nevertheless, plasma branched-chain keto-acids appear to represent the most sensitive signature of obesity (40). In contrast, Sun et al. showed that plasma leucine levels where decreased in the obese PCOS subgroup when compared with non-obese PCOS women (26). Also, leucine uptake differs throughout the follicle development, but metabolomics studies involving lean PCOS and lean controls showed no differences between groups regarding leucine (40). Leucine regulation of granulosa cell metabolism is important for understanding the role of environmental substrates, such as dietary leucine intake from protein rich foods in fertility regulation. Studies performed on mice showed that increased follicular influx of leucine increased follicular maturation. This is mediated via mTOR signaling activation (40). Also, leucine has been shown to reduce insulin resistance via the same mTOR pathway activation (40). Thus, increasing dietary leucine intake could improve insulin sensitivity and restore many metabolic abnormalities.

Altogether, the high variability of metabolomic signatures may, in fact, reflect the phenotypic heterogeneity of PCOS (27). This disorder can have a mild presentation in some individuals, mainly in lean women, while in others, particularly in obese women, a severe disturbance of endocrine and metabolic functions may occur (38). Due to this fact it is difficult to provide a unique metabolomic fingerprint to characterize PCOS.

Moreover, Zhao Y. et al. found that the decreased ratio of BCAA to aromatic AA (AAA) was directly correlated with the development of PCOS (38). This ratio was not influenced by obesity, nor by IR. However, serum levels of serine and threonine, but also valine and glycine, were closely correlated with both IR and obesity (38). Increased glycolysis may represent the key factor for increased serine serum levels in anovulatory PCOS patients. Specifically, ovulatory PCOS subtypes had reduced concentrations 
of serine and threonine, presumably because of the negative correlations of serine and threonine with obesity and IR. The decreased BCAA/AAA ratio in anovulatory patients was strongly related to the severity of the PCOS phenotypes, with the highest values in phenotype A (menstrual disorder + clinical and/or biochemical hyperandrogenism + polycystic ovaries) and lowest values in phenotype D (clinical and/or biochemical hyperandrogenism + polycystic ovaries) (38). Consequently, this ratio may be considered the hallmark of metabolic heterogeneity associated with PCOS (38). Increased levels of valine and decreased levels of glycine progressively changed as insulin sensitivity decreased in PCOS women compared with controls (43). Low levels of glycine downregulate adiponectin levels, which increases inflammation observed in PCOS (43). AAA levels and BCAA/AAA ratio changes were not induced by IR and obesity, but were strongly associated with PCOS severity (38). From this point of view, decreased BCAA/AAA ratio may play an important role in the development of PCOS (38).

Zhao X. et al. found increased levels of glycated phenylalanine in PCOS women as a consequence of accumulation of advanced glycation end products due to altered glucose and hormone metabolism (25). Moreover, the authors included glycated phenylalanine into a combined metabolite biomarker, together with dihydrotestosterone sulfate, uridine and FFA 18:1/FFA 18:0, FFA 20:3, which showed good sensitivity and specificity in PCOS screening (25). The validation process of this combination of biomarkers offered a 0.874 AUC and even higher for lean PCOS women (0.906 AUC) (24). These metabolites could provide a deeper insight in the pathogenesis of PCOS (24).

Recent studies also show increased levels of amino acids in obese women with PCOS when compared with metabolically healthy obese. These changes involve amino acids like gluta- mate, ornithine, tyrosine, methionine, proline, phenylalanine, and leucine/isoleucine. However, the authors demonstrated the positive impact of exercise on these metabolic alterations. After 8 weeks of supervised physical exercises, the levels of amino acids that were increased at baseline did not differ between PCOS and controls, despite no weight changes in both groups (44).

\section{Alterations of the tricarboxylic acid cycle (TCA) metabolism in obese PCOS women} Under fasting conditions, fatty acids, BCAA, and AAA are degraded to acetyl-CoA, which serves as a substrate in the tricarboxylic acid (TCA) cycle. The metabolite $\alpha$-ketoglutarate (also known as 2-oxoglutarate, 2-ketoglutaric acid, or oxoglutaric acid) is a key intermediate of TCA cycle. Zhao H. et al. showed that $\alpha$-ketoglutarate was significantly higher in the follicular fluid from obese women with PCOS in comparison with lean women with PCOS (27). Plasma $\alpha$-ketoglutarate was reported to be also associated with non-alcoholic fatty liver disease (NAFLD), a condition frequently associated with obesity (45).

Nonetheless, obese women with PCOS had a lower concentration of serum citrate versus non-obese patients (26). The decrease in citrate seems to be due to mitochondrial metabolism remodeling during adipocyte hypertrophy (26). However, this process could be an adaptation to prevent excessive influx of carbon into the Krebs cycle, reconfirming that during obesity there is a metabolic shift from energy provision to lipid storage. High levels of succinate, malate, and oxaloacetate and low levels of cis-aconitate in the follicular fluid of women with classic PCOS can decrease the mitochondrial biogenesis in the cumulus cells, but also can affect the developmental potential of oocytes $(27,33)$.

Moreover, Troisi et al. identified several other metabolites related to TCA cycle with altered concentrations: citric acid, glutamine, choline, 
and arginine were decreased, but valine and phenylalanine were increased (20). These modifications are also associated with oocyte maturation and insulin resistance $(45,46)$.

\section{Steroid metabolites profiling in patients with PCOS and obesity}

Wang et al. identified 3 urinary metabolites that were increased in obese PCOS women compared with the obese control group: cortolone-3-glucuronide, tetrahydroaldosterone-3-glucuronide, and testosterone glucuronide (37). Of these, cortolone-3-glucuronide and tetrahydroaldosterone-3-glucuronide correlated positively to obesity and triglycerides (37). Apparently, increased levels of these two metabolites are caused by altered glucuronidation, which occurs primarily in the liver. Moreover, both obesity and hypertriglyceridemia are associated with increased levels of circulating FFAs and increased prevalence of NAFLD, which could further increase the expression of the first two metabolites mentioned (48).

The best single hormonal marker, elevated in $86 \%$ of the women with PCOS, is testosterone glucuronide (37). Unlike plasma testosterone, urinary testosterone glucuronide is mostly independent of its short-term of circadian changes in the blood and it could be used as an indicator of androgen excess. Besides this, elevated testosterone glucuronide excretion and decreased urinary $11 \alpha$-hydroxyprogesterone were confirmed and validated as urinary biomarkers in patients with PCOS (37).

\section{Strengths and limitations}

To the best of our knowledge, this is the first systematic review to assess the impact of obesity on the metabolic fingerprints of PCOS women compared with controls. Even if we performed a comprehensive search of the literature, the number of articles included is low. This is due to the small number of studies overall that have focused on the topic chosen. Metabolomics is a new field to be discovered in different diseases in order to better understand their mechanism and act accordingly. From this point of view there is need for high-quality studies, properly controlled for potential confounders (i.e. obesity, insulin resistance, diabetes mellitus, etc.) and with longer follow-up period.

\section{Conclusions}

There is compelling evidence of significant alterations in carbohydrate, lipid, and amino acid metabolism among women with obesity-associated PCOS. Due to its phenotypic and pathophysiological heterogeneity, there is no typical metabolomic profile or single biomarker to predict PCOS. However, further metabolomic studies may provide crucial steps in identifying the metabolic pathways involved in PCOS.

\section{Abbreviations}

AA - amino-acids

AAA - aromatic AA

BCAA - branched-chain amino acids

BMI - body mass index

FFAs - free fatty acids

GC-MS - gas chromatography-mass spectrometry

HDL - High density lipoproteins - cholesterol

IR - insulin resistance

LC - liquid chromatography

LDL - Low density lipoproteins-cholesterol

LPC - lysophosphatidylcholines

MS - mass spectrometry

NAFLD - non-alcoholic fatty liver disease

NMR - nuclear magnetic resonance

PCOS - polycystic ovary syndrome

TCA - tricarboxylic acid

WHO - World Health Organization 


\section{Authors contributions}

Conceived and designed the review: CLV; Performed the search of articles: GR; AAM; Read all the articles and applied the exclusion criteria: AF; Substantial contribution to interpretation of data: SC; Gathered the information and valid biomarkers from the articles: ADF; Wrote the manuscript: CLV, CEG. All authors critically revised the manuscript for important intellectual content, approved the final the version to be submitted and agreed to be accountable for the content of the manuscript.

\section{Conflict of interest}

The authors report no conflict of interest related to the development of the present manuscript.

\section{References}

1. Conway G, Dewailly D, Diamanti-Kandarakis E, Escobar-Morreale HF, Franks S, Gambineri A et al. The polycystic ovary syndrome: a position statement from the European Society of Endocrinology. European Journal of Endocrinology 2014; 4 1-29. DOI: 10.1530/ EJE-14-0253

2. Wang R, Mol BW. The Rotterdam criteria for polycystic ovary syndrome: evidence-based criteria? Hum Reprod. 2017 Feb; 32(2):261-4. DOI: 10.1093/humrep/ dew287

3. Gambineri A, Pelusi C, Vicennati V, Pagotto U, Pasquali R. Obesity and the Polycystic Ovary Syndrome. Int J Obes Relat Metab Disord. 2002 Jul;26(7):883-96. DOI: 10.1038/sj.ijo.0801994

4. Sha, T, Wang X, Cheng W, Yan Y. A meta-analysis of pregnancy-related outcomes and complications in women with polycystic ovary syndrome undergoing IVF. Reprod Biomed Online. 2019 Aug;39(2):281-93. DOI: 10.1016/j.rbmo.2019.03.203

5. De Leo V, Musacchio MC, Cappelli V, Massaro MG, Morgante G, Petraglia F. Genetic, hormonal and metabolic aspects of PCOS: an update. Reprod Biol Endocrinol. 2016 Jul 16;14(1):38. DOI: 10.1186/s12958016-0173-x

6. Haoula Z, Ravipati S, Stekel DJ, Ortori CA, Hodgman
C, Daykin C et al. Lipidomic analysis of plasma samples from women with polycystic ovary syndrome Metabolomics. 2015;11(3):657-66. DOI: 10.1007/s11306014-0726-y

7. Dong F, Deng D, Chen H, Cheng W, Li Q, Luo R et al. Serum metabolomics study of polycystic ovary syndrome based on UPLC-QTOF-MS coupled with a pattern recognition approach. Anal Bioanal Chem. 2015 Jun;407(16):4683-95 DOI: 10.1007/s00216-0158670-X

8. Vonica CL, Fodor A, Roman G, Mureasan AA, Farcas $\mathrm{AD}$, Inceu $\mathrm{GV}$, et al. Metabolic Analysis in Women with Polycystic Ovary Highlight Specific Biomarkers of Insulin Resistance. Rev.Chim.(Bucharest). 2019;70(10):3644-8 DOI: 10.37358/RC.19.10.7613

9. Bellver J, De Los Santos MJ, Alamá P, Castelló D, Privitera L, Galliano D et al. Day-3 embryo metabolomics in the spent culture media is altered in obese women undergoing in vitro fertilization. Fertil Steril. 2015 Jun;103(6):1407-15.e1 DOI: 10.1016/j.fertnstert.2015.03.015

10. Vonica CL, Ilie IR, Socaciu C, Moraru C, Georgescu B, Farcaş A et al. Lipidomics biomarkers in women with polycystic ovary syndrome (PCOS) using ultra-high performance liquid chromatography-quadrupole time of flight electrospray in a positive ionization mode mass spectrometry. Scand J Clin Lab Invest. 2019 Oct;79(6):437-42. DOI: 10.1080/00365513.2019.1658215

11. Johnson CH, Ivanisevic J, Siuzdak G. Metabolomics: beyond biomarkers and towards mechanisms. Nat Rev Mol Cell Biol. 2016 Jul;17(7):451-9. DOI: 10.1038/ nrm.2016.25

12. Kosmides AK, Kamisoglu K, Calvano SE, Corbett SA, Androulakis IP. Metabolomic Fingerprinting: Challenges and Opportunities. Crit Rev Biomed Eng. 2013;41(3):205-21. DOI: 10.1615/CritRevBiomedEng.2013007736

13. Zhou B, Feng Xiao J, Tuli L. LC-MS-based metabolomics. Mol Biosyst. 2012 Feb; 8(2): 470-81. DOI: 10.1039/C1MB05350G

14. Lee K-M, Jeon J-Y, Lee B-J, Lee H, Choi H-K. Application of Metabolomics to Quality Control of Natural Product Derived Medicines. Biomol Ther (Seoul). 2017;25(6):559-68. DOI: 10.4062/biomolther.2016.249

15. Moher D, Liberati A, Tetzlaff J, Altman DG, Group P. Preferred reporting items for systematic reviews 
and meta-analyses: the PRISMA statement. J Clin Epidemiol. 2009;62:1006-12. DOI: 10.1016/j.jclinepi.2009.06.005

16. Slim K, Nini E, Forestier D, Kwiatkowski F, Panis Y, Chipponi J. Methodological index for non-randomized studies (minors): development and validation of a new instrument. ANZ J Surg. 2003;73: 712-6. DOI: 10.1046/j.1445-2197.2003.02748.x

17. Higgins JPT SJ, Savović J, Page MJ, Hróbjartsson A, Boutron I, Reeves B, Eldridge S. A revised tool for assessing risk of bias in randomized trials. In: Chandler J, McKenzie J, Boutron I, Welch V, editors. Cochrane Methods. Cochrane Database of Systematic Reviews 2016, Issue 10 (Suppl 1).

18. National Heart, Lung and blood institute. Quality Assessment Tool for Observational Cohort and Cross-Sectional Studies https://www. Int J Diabetes Dev Ctries Author's personal copy nhlbi.nih.gov/health-topics/ study-quality-assessment-tools. Accessed 28 Mar 2019.

19. Escobar-Morreale HF, Samino S, Insenser M, Vinaixa M, Luque-Ramírez M, Lasunción MA et al. Metabolic heterogeneity in polycystic ovary 846 syndrome is determined by obesity: plasma metabolomic approach using GC- 847 MS. Clin. Chem. 2012; 58, 999-1009. DOI: $10.1373 /$ clinchem.2011.176396

20. Troisi J, Cinque C, Giugliano L, Symes S, Richards $\mathrm{S}$, Adair D. Metabolomic change due to combined treatment with myo-inositol, D-chiro-inositol and glucomannan in polycystic ovarian syndrome patients: a pilot study. J Ovarian Res. 2019 Mar 23;12(1):25. DOI: 10.1186/s13048-019-0500-x

21. Goodpaster BH, Wolfe RR, Kelley DE. Effects of obesity on substrate utilization during exercise. Obesit Res. 2002;10(7):575-84. DOI: 10.1038/oby.2002.78

22. Kelley DE, Mandarino LJ. Fuel selection in human skeletal muscle in insulin resistance: A reexamination. Diabetes. 2000; 49(5):677-83. DOI: 10.2337/diabetes.49.5.677

23. Di Sarra D, Tosi F, Bonin C, Fiers T, Kaufman JM, Signori $\mathrm{C}$ et al. Metabolic inflexibility is a feature of women with polycystic ovary syndrome and is associated with both insulin resistance and hyperandrogenism. J Clin Endocrinol Metabol. 2013;98(6):2581-8. DOI: 10.1210/jc.2013-1161

24. Whigham LD, Butz DE, Dashti H, Tonelli M, Johnson LK, Cook ME et al. Metabolic Evidence of Diminished Lipid Oxidation in Women With Polycystic Ovary Syn- drome. Curr Metabolomics. 2014;2(4):269-78. DOI: 10 $.2174 / 2213235 X 01666131203230512$

25. Zhao X, Xu F, Qi B, Hao S, Li Y, Li Y et al. Serum Metabolomics Study of Polycystic Ovary Syndrome Based on Liquid Chromatography-Mass Spectrometry. J. Proteome Res. 2014;13(2):1101-11 DOI: 10.1021/ pr401130w

26. Sun L, Hu W, Liu Q, Hao Q, Sun B, Zhang Q et al. Metabonomics reveals plasma metabolic changes and inflammatory marker in polycystic ovary syndrome patients. J Ovarian Res. 2019 Mar 23;12(1):25.

27. Zhao H, Zhao Y, Li T, Li M, Li J, Li R et al. Metabolism alteration in follicular niche: the nexus between intermediary metabolism, mitochondrial function, and classic polycystic ovary syndrome. Free Radic Biol Med. 2015 Sep;86:295-307. DOI: 10.1016/j.freeradbiomed.2015.05.013

28. Attie AD, Krauss RM, Gray-Keller MP, Brownlie A, Miyazaki M, Kastelein JJ et al. Relationship between stearoyl-CoA desaturase activity and plasma triglycerides in human and mouse hypertriglyceridemia. J. Lipid Res. 2002, 43 (11), 1899-1907. DOI: 10.1194/ jlr.M200189-JLR200

29. Ntambi JM. The regulation of stearoyl-CoA desaturase (SCD). Prog. Lipid Res. 1995, 34 (2), 139-150. DOI: 10.1016/0163-7827(94)00010-J

30. Dobrzyn, A, Ntambi JM. The role of stearoyl-CoA desaturase in body weight regulation. Trends Cardiovasc. Med. 2004, 14 (2), 77-81. DOI: 10.1016/j. tcm.2003.12.005

31. Brown JM, Chung, S, Sawyer JK, Degirolamo C, Alger HM, Nguyen TM et al. Combined therapy of dietary fish oil and stearoyl-CoA desaturase 1 inhibition prevents the metabolic syndrome and atherosclerosis. Arterioscler Thromb Vasc Biol. 2010 Jan;30(1):24-30. DOI: 10.1161/ATVBAHA.109.198036

32. Mar-Heyming R, Miyazaki M, Weissglas-Volkov D, Kolaitis NA, Sadaat N, Plaisier C et al. Association of stearoyl-CoA desaturase 1 activity with familial combined hyperlipidemia. Arterioscler Thromb Vasc Biol. 2008 Jun;28(6):1193-9. DOI: 10.1161/ATVBAHA.107.160150

33. Perez-Chacon G, Astudillo AM, Ruiperez V, Balboa MA, Balsinde J. Signaling role for lysophosphatidylcholine acyltransferase 3 in receptor-regulated arachidonic acid reacylation reactions in human monocytes. J. Immunol. 2010; 184 (2), 1071-8. DOI: 10.4049/jim- 
munol.0902257

34. Barber MN, Risis S, Yang C, Meikle PJ, Staples M, Febbraio MA et al. Plasma Lysophosphatidylcholine Levels Are Reduced in Obesity and Type 2 Diabetes. PLoS One. 2012;7(7):e41456. DOI: 10.1371/journal. pone.0041456

35. Chang AY, Lalia AZ, Jenkins GD, Dutta T, Carter RE, Singh RJ et al. Combining a Nontargeted and Targeted Metabolomics Approach to Identify Metabolic Pathways Significantly Altered in Polycystic Ovary Syndrome. Metabolism. 2017 Jun;71:52-63. DOI: 10.1016/j.metabol.2017.03.002

36. Diamanti-Kandarakis E, Dunaif A. Insulin resistance and the polycystic ovary syndrome revisited: an update on mechanisms and implications. Endocr Rev. 2012 Dec; 33(6):981-1030. DOI: 10.1210/er.2011-1034

37. Wang W, Wang S, Tan S, Wen M, Qian Y, Zeng X et al. Detection of urine metabolites in polycystic ovary syndrome by UPLC triple-TOF-MS. Clin Chim Acta. 2015 Aug 25;448:39-47. DOI: 10.1016/j.cca.2015.06.008

38. Zhao Y, Fu L, Li R, Wang LN, Yang Y, Liu NN et al. Metabolic profiles characterizing different phenotypes of polycystic ovary syndrome: plasma metabolomics analysis. BMC Medicine. 2012 Nov 30;10:153. DOI: 10.1186/1741-7015-10-153

39. Wang TJ, Larson MG, Vasan RS, Cheng S, Rhee EP, McCabe E et al. Metabolite profiles and the risk of developing diabetes. Nat Med. 2011 Apr;17(4):448-53. Epub 2011 Mar 20. DOI: 10.1038/nm.2307

40. Martin FP, Montoliu I, Collino S, Scherer M, Guy P, Tavazzi I, et al. Topographical body fat distribution links to amino acid and lipid metabolism in healthy non-obese women. LoS One. 2013 Sep 11;8(9):e73445. DOI: 10.1371/journal.pone.0073445

41. Chand AL, Legge M. 2011. Amino acid transport system $\mathrm{L}$ activity in developing mouse ovarian follicles.
Hum Reprod. 2011 Nov;26(11):3102-8. DOI: 10.1093/ humrep/der298

42. She P, Olson KC, Kadota Y, Inukai A, Shimomura Y, Hoppel CL, et al Leucine and protein metabolism in obese Zucker rats, PLoS One. 2013;8(3):e59443.. DOI: 10.1371/journal.pone.0059443

43. Li X, Wang Y, Tang W, Liu C, Chen Y, Zheng Z. Relationship between ADIPOQ polymorphisms and polycystic ovary syndrome: a meta-analysis involving 6,735 subjects. Int J Clin Exp Med 2017;10(2):1828-39.

44. Halama A, Aye MM, Dargham SR., Kulinski M, Suhre $\mathrm{K}$, Atkin SL. Metabolomics of Dynamic Changes in Insulin Resistance Before and After Exercise in PCOS. Front Endocrinol (Lausanne). 2019 Feb 27;10:116. DOI: $10.3389 /$ fendo.2019.00116

45. Rodríguez-Gallego E, Guirro M, Riera-Borrull M, Hernández-Aguilera A, Mariné-Casadó R, Fernández-Arroyo S, et al. Mapping of the circulating metabolome reveals $\alpha$-ketoglutarate as a predictor of morbid obesity-associated non-alcoholic fatty liver disease. Int J Obes (Lond). 2015 Feb;39(2):279-87. DOI: 10.1038/ ijo. 2014.53

46. Gaster M, Nehlin JO, Minet AD. Impaired TCA cycle flux in mitochondria in skeletal muscle from type 2 diabetic subjects: marker or maker of the diabetic phenotype? Arch Physiol Biochem. 2012 Jul;118(3):156-89. DOI: 10.3109/13813455.2012.656653

47. Jimenez PT, Frolova AI, Chi MM, Grindler NM, Willcockson AR, Reynolds KA, et al. DHEA-mediated inhibition of the pentose phosphate pathway alters oocyte lipid metabolism in mice. Endocrinology. 2013 Dec;154(12):4835-44. DOI: 10.1210/en.2012-2140

48. Guan HP, Goldstein JL, Brown MS, Liang G. Accelerated fatty acid oxidation in muscle averts fasting-induced hepatic steatosis in SJL/J mice. J Biol Chem. 2009 Sep 4;284(36):24644-52. DOI: 10.1074/jbc.M109.034397 